\title{
Inhomogeneous and anisotropic equilibrium state of a swollen hydrogel containing a hard core
}

\author{
Xuanhe Zhao, Wei Hong, and Zhigang Suo ${ }^{\text {a) }}$ \\ School of Engineering and Applied Sciences, Harvard University, Cambridge, MA 02138
}

\begin{abstract}
A polymer network can imbibe water from environment and swell to an equilibrium state. If the equilibrium is reached when the network is subject to external mechanical constraint, the deformation of the network is typically anisotropic, and the concentration of water inhomogeneous. Such an equilibrium state in a network constrained by a hard core is modeled here with a nonlinear differential equation. The presence of the hard core markedly reduces the concentration of water near the interface and causes high stresses.
\end{abstract}

Keywords: core-shell, hydrogel, inhomogeneous and anisotropic swelling

a) suo@seas.harvard.edu 
A network of flexible polymers can imbibe water from environment and swell, often to a volume many times the initial volume of the network. Swelling is mainly an entropic process: the sorption of water increases the number of configurations of the mixture, but the expansion of the network reduces the number of configurations of the network. ${ }^{1}$ The competing trends equilibrate water molecules in the network with those in the environment. The resulting aggregate of polymers and water molecules is known as a polymeric hydrogel.

When a network equilibrates with environment under no external mechanical constraint, the network swells by a homogeneous and isotropic expansion, and water molecules distribute in the gel homogeneously. When the network equilibrates with environment under external mechanical constraint, however, the network may swell by an inhomogeneous and anisotropic deformation, and water molecules may distribute in the gel inhomogeneously. Such inhomogeneous and anisotropic equilibrium states are common, because mechanical constraint is ubiquitous in applications of gels. Yet few theoretical analyses exist for gels in inhomogeneous and anisotropic equilibrium states, possibly due to the mathematical difficulty associated with large deformation and nonlinear equations.

This paper studies a relatively simple structure: a spherical shell of gel surrounding a hard core of another material. Such core-shell structures have been intensely studied experimentally in recent years $^{2-4}$. Potential applications include drug delivery ${ }^{5-8}$, controlled self-assembly ${ }^{9,10}$, medical devices ${ }^{11}$, photonic crystals $^{12,13}$, and micro-actuators ${ }^{14}$. In many applications, an understanding of the equilibrium distribution of water is of essential importance. Also important is the distribution of stresses, as debonding between the gel and the core has 
been reported ${ }^{2,15}$. Here we describe the inhomogeneous and anisotropic equilibrium state by formulating a nonlinear differential equation.

Fig. 1 illustrates a core-shell structure. A water-free and stress-free polymer network is taken as the reference state, Fig. 1(a), where the network is a hollow spherical shell, with inner radius $A$ and outer radius $B$. In the equilibrium state, Fig. 1(b), the network is swollen and contains a hard core of radius $A \lambda_{0}$. The initial stretch, $\lambda_{0}$, is set by the method to prepare the structure. For example, the structure can be prepared by first coating the core with a shell of an aqueous solution, and then cross-linking the polymers in the solution. In this case, $\lambda_{0}$ relates to the volume fraction of water in the initial solution.

The structure is subsequently immersed in pure water to swell further. After some time, water molecules in the gel equilibrate those in pure water, and the gel swells to an inhomogeneous and anisotropic state. This behavior is qualitatively understood as follows. The core is taken to be rigid and bonded to the network, so that near the interface the network cannot further stretch in the circumferential directions, and is constrained to expand only in the radial direction. Away from the interface, however, the network can stretch in all three directions. Consequently, the concentration of water in the gel is expected to be low near the interface, and increases away from the interface.

The inhomogeneous and anisotropic equilibrium state in an absorbent was formulated by Gibbs $^{16}$. He described large deformation with deformation gradient, mechanical equilibrium with differential equations involving nominal stresses, and chemical equilibrium with a uniform chemical potential of the solvent. Gibbs derived the equations of state formally from a 
free-energy density, which is a function of the deformation gradient and the solvent concentration. However, he did not give any explicit form of this function. His theory has reappeared in most subsequent studies; see Refs ${ }^{17-22}$ for recent contributions. Here we will follow the notation of our recent paper ${ }^{22}$, and adopt the free-energy function introduced by Flory and Rehner ${ }^{23}$.

With reference to Fig. 1, imagine each element of the network is attached with a marker, which moves as the network deforms. The marker is at distance $R$ from the center in the reference state, and is at distance $r$ from the center in the equilibrium state. The deformed network is taken to retain the spherical symmetry, so that the deformation is fully specified by the function $r(R)$. Markers on a spherical surface of radius $R$ in the reference state move to a spherical surface of radius $r$ in the equilibrium state. Consequently, the stretch in every circumferential direction is

$$
\lambda_{\theta}=r / R
$$

Two nearby markers in a radial direction, of positions $R$ and $R+d R$ in the reference state, move to positions $r(R)$ and $r(R+d R)$ in the equilibrium state. The distance between the two markers is $d R$ in the reference state, and is $r(R+d R)-r(R)=d r$ in the equilibrium state, so that the stretch in the radial direction is

$$
\lambda_{r}=d r / d R
$$

An element of the network, of unit volume in the reference state, swells to volume $\lambda_{\theta}^{2} \lambda_{r}$ in the equilibrium state. Individual polymers and water molecules are taken to be incompressible, so that the change in volume of the gel is due to imbibing water molecules, 


$$
v C=\lambda_{\theta}^{2} \lambda_{r}-1
$$

where $v$ is the volume per solvent molecule, and $C$ is the concentration of water in the gel (i.e., the number of water molecules in an element of the gel in the equilibrium state divided by the volume of the element in the reference state).

In the equilibrium state, the gel develops a field of stress. Let $s_{\theta}(R)$ be the nominal stress in each circumferential direction, and $s_{r}(R)$ be the nominal stress in the radial direction. Mechanical equilibrium requires that

$$
\frac{d s_{r}}{d R}+2 \frac{s_{r}-s_{\theta}}{R}=0 .
$$

The chemical potential of water molecules in pure water is set to be zero. Consequently, the chemical potential of water molecules in the gel is also zero in the equilibrium state. Using the free energy of Flory and Rehner ${ }^{23}$, we write the equations of state ${ }^{22}$ :

$$
\begin{gathered}
\frac{s_{\theta}}{N k T}=\lambda_{\theta}-\lambda_{\theta}^{-1}+\frac{\lambda_{\theta} \lambda_{r}}{v N}\left[\log \frac{v C}{1+v C}+\frac{1}{1+v C}+\frac{\chi}{(1+v C)^{2}}\right], \\
\frac{s_{r}}{N k T}=\lambda_{r}-\lambda_{r}^{-1}+\frac{\lambda_{\theta}^{2}}{v N}\left[\log \frac{v C}{1+v C}+\frac{1}{1+v C}+\frac{\chi}{(1+v C)^{2}}\right],
\end{gathered}
$$

where $N$ is the number of polymer chains divided by the volume of the dry network, $k T$ is the temperature in the unit of energy, and $\chi$ is a dimensionless measure of the enthalpy of mixing. In practice, $v N$ is in the range $10^{-2} \sim 10^{-5}$, and $\chi$ is in the range $0.1 \sim 0.5$ for good solvents.

As a special case, when the network swells freely under no external mechanical constant, in equilibrium, the network undergoes a homogeneous and isotropic expansion. Setting $\lambda_{r}=\lambda_{\theta}=\lambda_{\text {free }}=\left(v C_{\text {free }}+1\right)^{1 / 3}$ and $s_{\theta}=0$ in (5), we obtain a nonlinear algebraic equation that 
determines the equilibrium concentration of water in the free-swelling gel, $v C_{\text {free }}$.

When the network swells subject to the constraint of the hard core, a combination of (1)-(6) leads to a nonlinear second-order differential equation that governs the function $r(R)$. This equation is solved numerically, subject to the boundary conditions $r(A)=\lambda_{0} A$ and $s_{r}(B)=0$.

Fig. 2 (a) plots the equilibrium concentration of water, $v C$, i.e., the ratio of the volume of water in the equilibrium state to the volume of the dry network. The equilibrium concentration of water in the gel is inhomogeneous: $\sim 12$ near the interface and $\sim 28$ at the outer surface. The latter is close to the equilibrium concentration of water in a free-swelling gel, $v C_{\text {free }}$, which is marked as the dashed line in Fig. 2(a). Evidently the effect of the constraint on the gel is localized within a radius only slightly larger than the radius of the core. The inhomogeneous distribution of water in a core-shell structure has been observed experimentally ${ }^{24}$. Fig. 2(b) plots the stretches in the gel. Near the interface, $\lambda_{\theta}$ is constrained by the core and does not change during swelling, but $\lambda_{r}$ increases substantially. Near the outer surface, both stretches approach that of a free-swelling gel, $\lambda_{\text {free }}$. Fig. 3(c) plots the stresses in the gel. Near the interface, $s_{\theta}$ is compressive, but $s_{r}$ is tensile. Near the outer surface, both stresses diminish. The magnitudes of the stresses are substantial, scaling with the elastic modulus of the dry network, $N k T$. The tensile radial stress may cause the gel to debond from the core ${ }^{2,15}$.

The outer radius $b$ of the gel in the equilibrium state is important in some applications. Fig. 3(a) plots $b / B$ as a function of $B / A$. When the network is thin (i.e., $B / A$ is small), nearly the entire volume of the network is affected by the core, so that $b / B \approx \lambda_{0}$. When the network is thick (i.e., $B / A$ is large), the network away from the interface is nearly unaffected by 
the core, so that $b / B \approx \lambda_{\text {free }}$. The gel swells less when the network is either more hydrophobic [i.e., large positive $\chi$ in Fig. 3(b)], or more densely corssliked [i.e., large $v N$ in Fig. 3(c)].

In summary, a polymer network swelling under external mechanical constraint typically attains an inhomogeneous, anisotropic equilibrium state. Our calculations show that, near the core-shell interface, the concentration of water is greatly reduced, and the stresses are high. These trends agree with available experimental observations. The inhomogeneous distribution of water in the equilibrium state clearly shows that diffusion in gels should not be analyzed using Fick's law, which assumes that the diffusion flux is proportional to the concentration gradient. Instead, more general kinetic laws should be invoked. ${ }^{17-22}$

This work is supported by the MRSEC, of Harvard University, funded by the National Science Foundation. 


\section{References}

1 P. J. Flory, Principles of Polymer Chemistry (Cornell University Press, Ithaca, 1953).

$2 \quad$ M. Ballauff and Y. Lu, Polymer 48, 1815-1823 (2007).

3 S. Nayak and L. A. Lyon, Angewandte Chemie-International Edition 44, 7686-7708 (2005).

$4 \quad$ R. Pelton, Advances in Colloid and Interface Science 85, 1-33 (2000).

5 L. Vodna, S. Bubenikova, and D. Bakos, Macromolecular Bioscience 7, 629-634 (2007).

$6 \quad$ H. Otsuka, Y. Nagasaki, and K. Kataoka, Advanced Drug Delivery Reviews 55, 403-419 (2003).

7 J. H. Kim and T. R. Lee, Drug Development Research 67, 61-69 (2006).

8 N. Sahiner, A. M. Alb, R. Graves, T. Mandal, G. L. McPherson, W. F. Reed, and V. T. John, Polymer 48, 704-711 (2007). H. Wei, X. Z. Zhang, Y. Zhou, S. X. Cheng, and R. X. Zhuo, Biomaterials 27, 2028-2034 (2006).

K. Nakahama and K. Fujimoto, Langmuir 18, 10095-10099 (2002). 


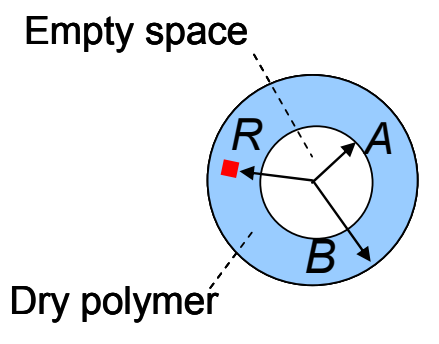

(a)

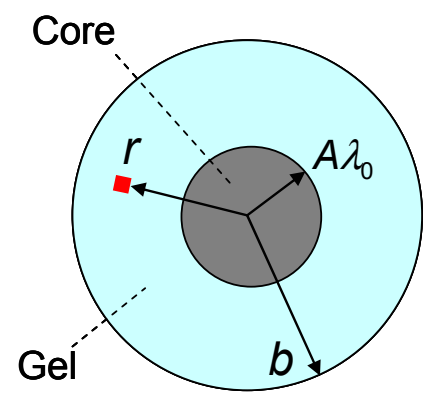

(b)

\section{Reference State}

\section{Equilibrium State}

FIG.1. (a) In the reference state, the polymer network is water-free and stress-free. (b) In the equilibrium state, the network is swollen and contains the rigid core. 

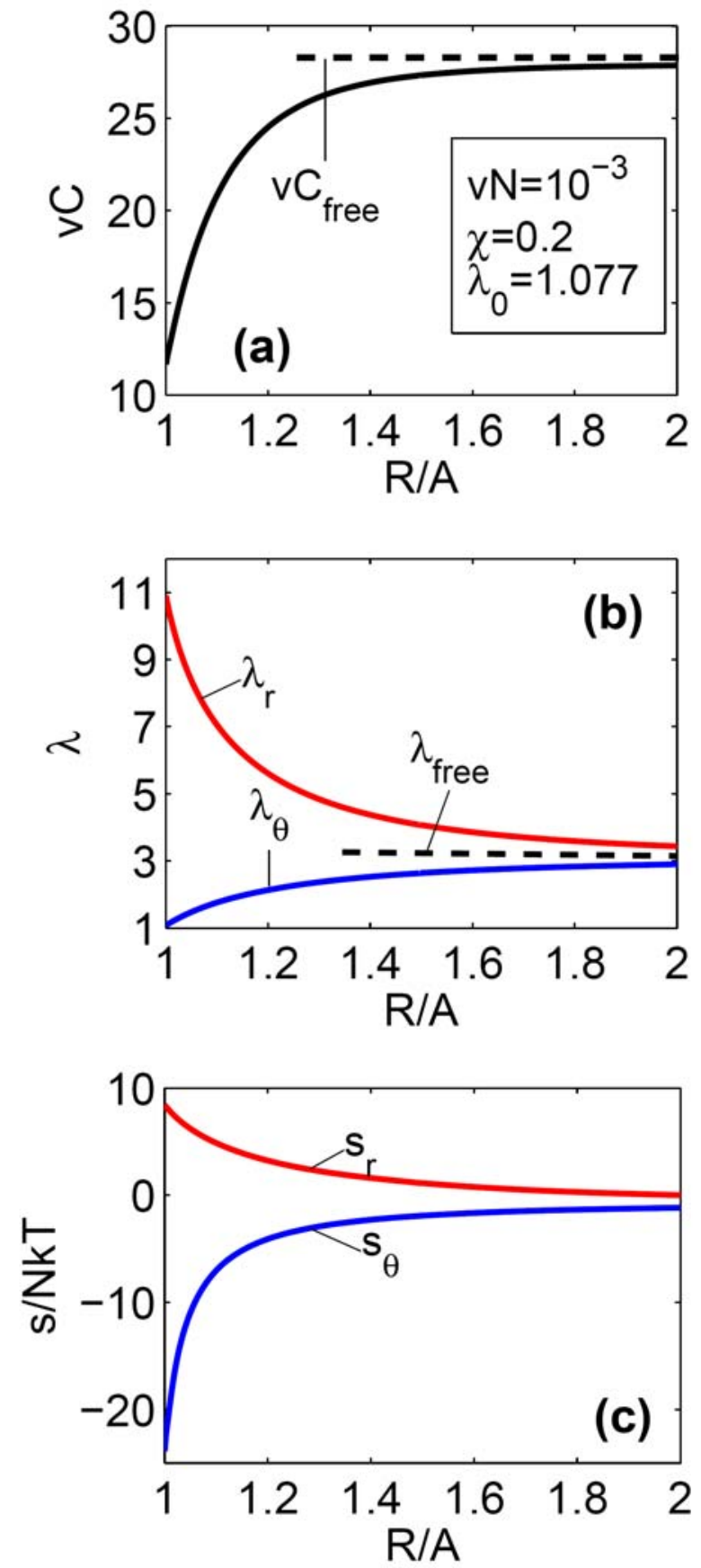

FIG. 2. The equilibrium distributions of (a) water concentration, (b) stretches, and (c) stresses. 

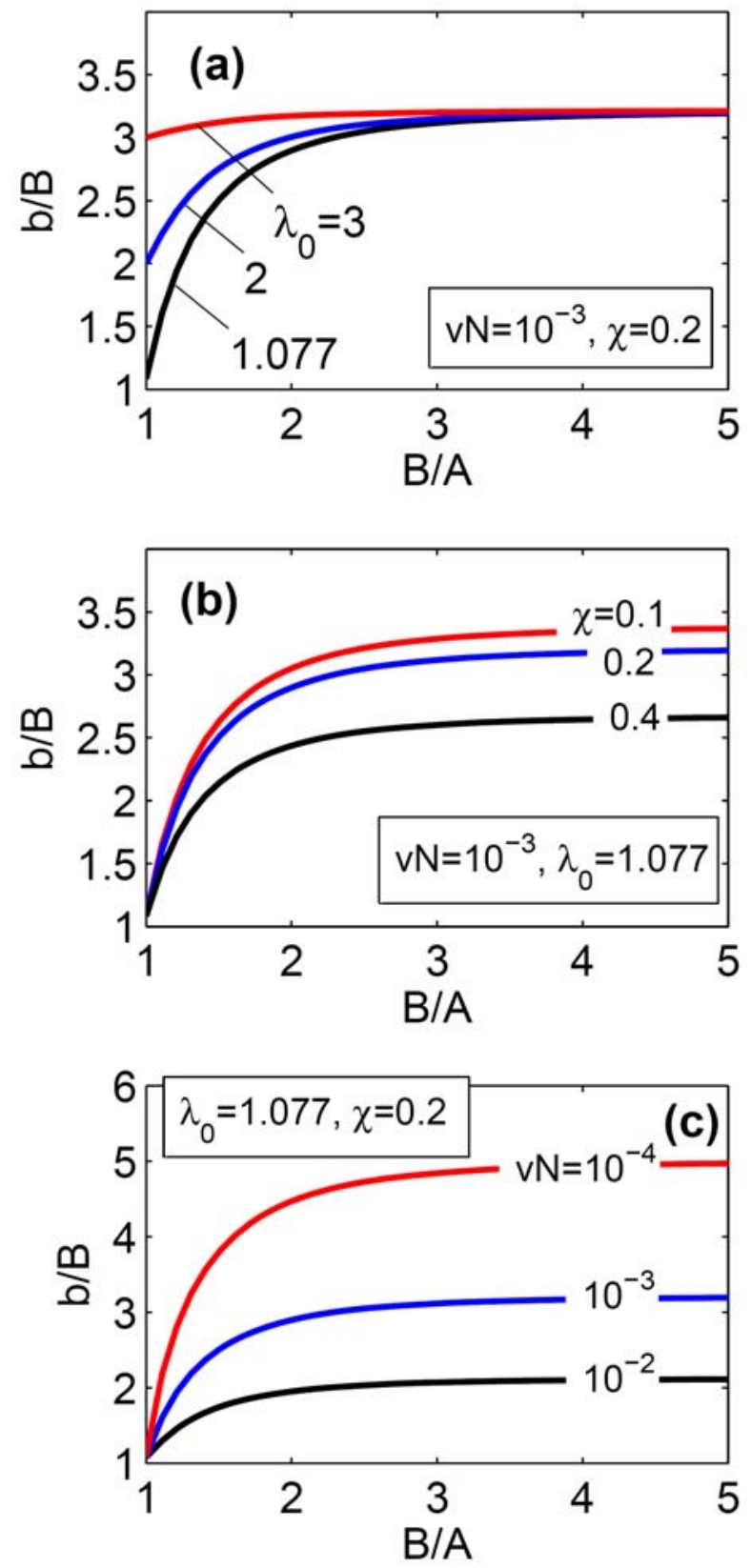

FIG. 3. Swelling ratio $b / B$ as a function of $B / A$ at various values of (a) $\lambda_{0},(b) \chi$, and (c) $v N$ 